\title{
Seoritae Extract Reduces Prostate Weight and Suppresses Prostate Cell Proliferation in a Rat Model of Benign Prostate Hyperplasia
}

\author{
Hoon Jang, ${ }^{1}$ Woong-Jin Bae, ${ }^{1}$ Seung-Mo Yuk, ${ }^{1}$ Dong-Seok Han, ${ }^{1}$ U-Syn Ha, ${ }^{1}$ \\ Seong-Yeon Hwang, ${ }^{2}$ Shin-Hee Yoon, ${ }^{3}$ Sae-Woong Kim, ${ }^{1}$ and Chang-Hee Han ${ }^{1,4}$ \\ ${ }^{1}$ Department of Urology, The Catholic University of Korea College of Medicine, Seoul St. Mary's Hospital, 222 Banpo-daero, \\ Seocho-Gu, Seoul 137-701, Republic of Korea \\ ${ }^{2}$ Korea Bio Medical Science Institute, Prime Tower 1st Floor Sinmunno 2-ga, Jongno-gu, Seoul 110-062, Republic of Korea \\ ${ }^{3}$ The Catholic Agro-Medical Center, The Catholic University of Korea, Seoul St. Mary's Hospital, 222 Banpo-daero, \\ Seocho-Gu, Seoul 137-701, Republic of Korea \\ ${ }^{4}$ The Catholic University of Korea, Uijeongbu, St. Mary's Hospital 271, Cheon Bo-Ro, Uijeongbu-Si, \\ Gyeonggi-Do 480-717, Republic of Korea \\ Correspondence should be addressed to Chang-Hee Han; urohan@catholic.ac.kr
}

Received 13 November 2013; Revised 12 January 2014; Accepted 13 January 2014; Published 19 February 2014

Academic Editor: Khalid Rahman

Copyright (c) 2014 Hoon Jang et al. This is an open access article distributed under the Creative Commons Attribution License, which permits unrestricted use, distribution, and reproduction in any medium, provided the original work is properly cited.

\begin{abstract}
Seoritae is a type of black soybean that is known to have health-promoting effects due to its high isoflavone and anthocyanin contents. We evaluated whether Seoritae extract (SE) had beneficial effects on the reduction of prostate weight in a rat model of benign prostatic hyperplasia (BPH). BPH was induced by intramuscular injections of testosterone enanthate once a week for 5 weeks in Sprague-Dawley rats, and rats were treated with or without daily oral doses of SE during BPH induction. After 5 weeks, the oxidative stress (superoxide dismutase and 8-hydroxy-2-deoxyguanosine), apoptosis (caspase-3), and activity of 5-alpha reductase were evaluated in the serum and prostate. The SE treatment group showed a significant decrease in prostate weight, oxidative stress, apoptosis, and 5-alpha reductase activity compared to the nontreated BPH group. These results show that SE is effective in decreasing the weight and proliferation of the prostate, and suggest that SE may be an effective treatment for BPH.
\end{abstract}

\section{Introduction}

The average life-span of human beings is gradually increasing due to development in science and medicine [1]. As a result, the elderly currently account for a significant portion of the population, and the quality of life in this age group has become a social concern. Benign prostate hyperplasia (BPH), which is the limitless growth of the prostate without malignancy, has been shown to have a high incidence in elderly males and affects their quality of life.

Although the molecular biological mechanisms influencing the etiology of $\mathrm{BPH}$ have not been elucidated; dihydrotestosterone (DHT), which is the more active from of testosterone and is converted from testosterone by 5 -alpha reductase, is known to play a central role in the prostate growth [2] and the oxidative stress-mediated mechanism is believed to also be associated with prostate cell hyperproliferation and tissue deformity $[3,4]$. In our previous report, we suggested that the oxidative stress mechanism is related to the occurrence and progression of $\mathrm{BPH}$ and that anthocyanin is a potent antioxidant that can decrease prostate volume and prevent $\mathrm{BPH}$ progression [5]. This study builds upon the work from our previous study by evaluating the relationship between oxidative stress and the occurrence and progression of BPH. In addition, we hypothesized that inhibition of 5alpha reductase would decrease the conversion of DHT from testosterone and antioxidant reactions that serve to reduce oxidative stress may prevent the occurrence and progression of $\mathrm{BPH}$. 
Seoritae is a type of black soybean (Glycine max (L.) Merr.) grown in Korea, which gets its name from the fact that it is harvested early in October when the first frost occurs. Unlike other black soybeans, the inside of Seoritae is of a bluish color. It is a traditional Korean food and is known to have health-promoting effects due to its high isoflavone and anthocyanin contents. Isoflavones have variable effects on growth factor inhibition [6], antioxidant properties [7, 8], cell adhesion [9], 5-alpha reductase activity [10, 11], and angiogenesis [12].

Anthocyanin is a known antioxidant that has antiangiogenic, anticarcinogenic, and antioxidant effects [13-15]. In particular, we hypothesized that the 5-alpha reductase inhibitory effect as well as the antioxidant properties of the isoflavones and anthocyanin in SE would be helpful in preventing the occurrence and progression of BPH.

Therefore, we administered Seoritae extract (SE) to rats during the induction of $\mathrm{BPH}$. Prostate weight, oxidative stress, apoptosis, and 5-alpha reductase activity were then measured in order to investigate the mechanism by which SE regulates oxidative stress and prostate cell proliferation so as to determine its potential in treating $\mathrm{BPH}$.

\section{Materials and Methods}

2.1. Preparation of SE. The SE used in our experiment was produced using the following method: Seoritae (1500 g) samples were extracted with $12,000 \mathrm{~mL}$ of $30 \%$ ethanol for $3 \mathrm{~h}$ at $90-100^{\circ} \mathrm{C}$. The solution was then filtered twice through a $50 \mu \mathrm{m}$ and a $1 \mu \mathrm{m}$ filter and concentrated in a vacuum evaporator $\left(60^{\circ} \mathrm{C}\right)$ to 70 brix. The residual solvent was removed from SE by using a drying machine for $18 \mathrm{~h}$ at $60^{\circ} \mathrm{C}$ in a vacuum. The resulting powder was then stored in plastic bag until use.

2.2. Analysis of Isoflavones and Anthocyanin from Seoritae Extracts. Isoflavones in SE were analyzed by highperformance liquid chromatography (HPLC) using a Waters 2695 Preparation Module HPLC system with a Waters 996 Photodiode Array Detector (Waters Corporation; Milford, MA, USA). Six peaks were obtained in the HPLC chromatogram by diode array detection (DAD) at $260 \mathrm{~nm}$. Major peaks $1,2,3,4,5$, and 6 were identified as daidzin, glycitin, genistin, daidzein, glycitein, and genistein, respectively, by comparison with HPLC retention times of our standard compounds (Figure 1(a)).

The anthocyanin content in SE was analyzed by HPLC using a Waters HPLC system with a 2487 Dual Wavelength Detector set at $520 \mathrm{~nm}$. Cyanidin-3-O-glucoside, one of the anthocyanins, was identified in the HPLC chromatogram by comparison with the HPLC retention times of our standard compounds (Figure 1(b)). The proportions of the isoflavones and anthocyanins in the SE are shown in Table 1.

2.3. Animal Groups and Treatment Protocol. Forty-eight 16week-old Sprague-Dawley male rats were treated under a protocol approved by the Institutional Animal Care and Use Committee (CUMC-2013-0117-01) and handled according to
TABLE 1: HPLC analysis of isoflavones and anthocyanin content $(\mathrm{mg} / \mathrm{g})$ of Seoritae extract. Triplicate samples of the Seoritae extract were analyzed by HPLC.

\begin{tabular}{lccc}
\hline Content & \multicolumn{3}{c}{ Seoritae extract } \\
\hline Daidzin & 3.171 & 4.146 & 4.861 \\
Glycitin & 0.287 & 0.369 & 0.438 \\
Genistin & 3.750 & 4.906 & 5.726 \\
Daidzein & 0.036 & 0.048 & 0.064 \\
Glycitein & 0.161 & 0.207 & 0.240 \\
Genistein & 0.043 & 0.055 & 0.073 \\
Total & $\mathbf{7 . 4 4 8}$ & $\mathbf{9 . 7 3 1}$ & $\mathbf{1 1 . 4 0 2}$ \\
\hline Anthocyanin & 0.120 & 0.122 & 0.124 \\
(Cyanidin-3-O-glucoside) & & &
\end{tabular}

National Institutes of Health (NIH) guidelines. Rats were divided equally into 4 groups ( $n=12$ each): control, $\mathrm{BPH}$, and $\mathrm{BPH}$ treated with SEs $(\mathrm{BPH}+\mathrm{SE} 1$ and $\mathrm{BPH}+\mathrm{SE} 2)$. To prevent the influence of intrinsic testosterone, all rats in the $\mathrm{BPH}$ and $\mathrm{BPH}+\mathrm{SE}$ groups underwent bilateral orchiectomies performed 3 days prior to the induction of $\mathrm{BPH}$. Prostate hyperplasia was induced in the $\mathrm{BPH}$ and $\mathrm{BPH}+$ SEs groups once a week by intramuscular injections of testosterone enanthate ( $25 \mathrm{mg}$; Rotexmedica $\mathrm{GmbH}$; Trittau, Germany) for 5 weeks. During BPH induction, rats in the $\mathrm{BPH}+\mathrm{SEs}$ groups were treated daily with oral SE at two concentrations (the $\mathrm{BPH}+\mathrm{SE} 1$ group received $228 \mathrm{mg} / \mathrm{kg}$ of SE and the $\mathrm{BPH}+\mathrm{SE} 2$ group received $457 \mathrm{mg} / \mathrm{kg}$ of SE), which were dissolved in $1 \mathrm{~mL}$ distilled water and administered orally through an $8 \mathrm{~F}$ red Rob-Nel catheter once a day for 5 weeks. The SE2 dose was the converted dosage for rats that represented the maximal suggested dosage of isoflavones for humans under the Korean Ministry of Food and Drug Safety (MFDS) guidelines. After 5 weeks, all rats were sacrificed. The blood was collected from the vena cava and the prostate was removed and weighed. The oxidative stress and the activity of 5-alpha reductase were analyzed in the serum and prostate, and apoptosis was assessed in the prostate samples.

2.4. Measurement of Oxidative Stress in Serum. To evaluate the oxidative stress in serum, we measured the total activity of superoxide dismutase (SOD). The total activity of SOD in serum was determined using a WST SOD assay kit (Dojindo Molecular Technologies; Kumamoto, Japan), which estimates SOD activity by measuring the inhibition of xanthin oxidase activity. Serum preparation for the total activity of SOD was performed according to the manufacturer's protocol. The optical density (OD) was determined at $450 \mathrm{~nm}$ using a microplate reader (Bio-Rad Model 550; CA, USA). The SODlike activity was calculated by using the following equation: SOD activity $=\{[($ A blank1 - A blank3 $)-($ A sample A blank2)]/(A blank1 - A blank3)\} $\times 100$.

2.5. Measurement of Oxidative Stress in the Prostate. Oxidative stress in the prostate was evaluated by quantifying the levels of 8 -hydroxy-2-deoxyguanosine $(8-\mathrm{OHdG})$ as a 


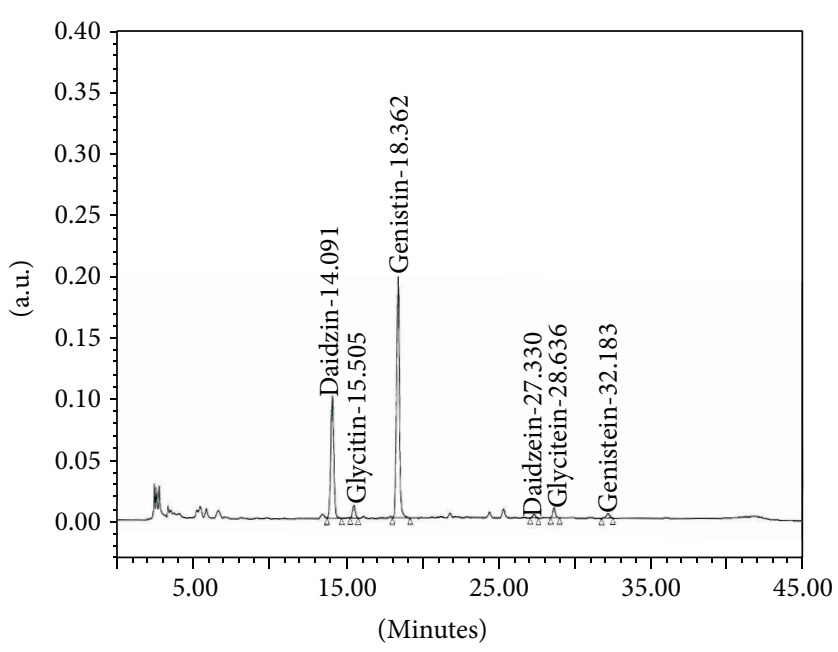

(a)

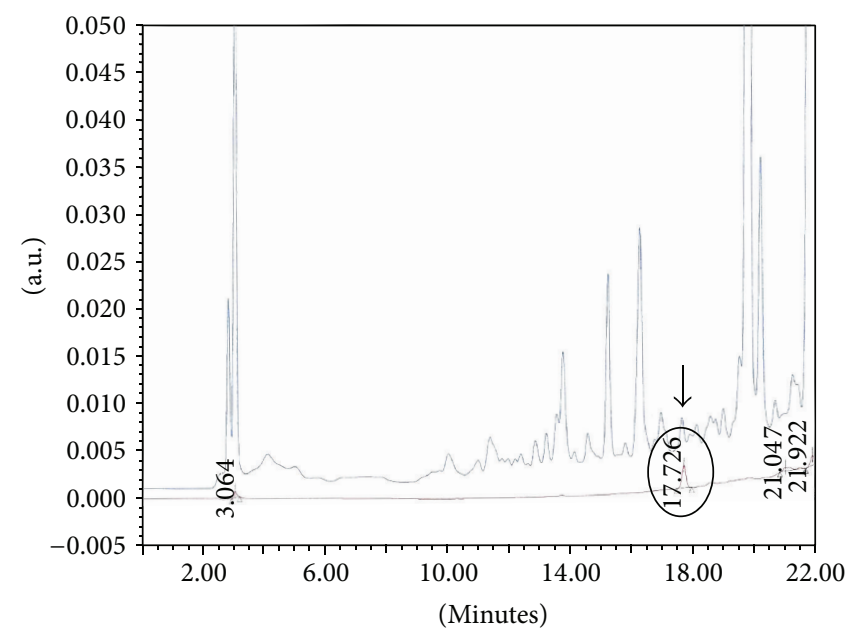

(b)

FIGURE 1: HPLC chromatogram of the Seoritae extract. (a) Six peaks were obtained and each peak represented the isoflavone content. (b) The peak with the circle indicates Cyanidin-3-O-glucoside in standard compounds. A corresponding peak was seen in the SE HPLC chromatogram (black arrow).

measurement of oxidatively modified DNA. Total DNA was extracted from the tissues using a DNeasy Blood and Tissue Kit (Qiagen, Valencia, CA), according to the manufacturer's instructions. The levels of $8-\mathrm{OHdG}$ were measured using a DNA oxidation kit (Highly Sensitive 8-OHdG Check ELISA; Japan Institute for the Control of Aging; Fukuroi, Japan), according to the manufacturer's protocol. The 8-OHdG standard (0.5-40 ng/mL) or 15-20 $\mu \mathrm{g}$ of DNA purified from the tissues was incubated for $1 \mathrm{~h}$ with a monoclonal antibody against $8-\mathrm{OHdG}$ in a microtiter plate precoated with 8 OHdG. After the color was developed with the addition of $3,3^{\prime}, 5,5^{\prime}$-tetramethylbenzidine, absorbance was measured at $450 \mathrm{~nm}$. Tissue sample concentration was calculated from a standard curve and was corrected for DNA concentration.

2.6. Measurement of Apoptosis. To assess apoptosis in the prostate, the concentration of caspase- 3 was measured using an ApoTarget Caspase-3/CPP32 Colorimetric Protease Assay Kit (Invitrogen; Camarillo, CA, USA). Tissue preparation for the quantification of caspase- 3 was performed according to the manufacturer's protocol. Samples were read at $400 \mathrm{~nm}$ in a microplate reader.

2.7. Measurement of 5-Alpha Reductase Activity. The activity of 5-alpha reductase was evaluated by the quantitative measurement of steroid 5-alpha reductase 2 (SRD5a2). In serum and prostate tissue, the SRD5a2 levels were measured in duplicate with SEM285Ra (USCN; Houston, TX, USA), a commercially available rat-specific enzyme-linked immunosorbent assay (ELISA) kit. Serum and tissue preparation was performed according to the manufacturer's protocol. Samples were read at $450 \mathrm{~nm}$ in a microplate reader.

2.8. Statistical Analysis. Data were analyzed statistically and expressed as the mean \pm SD. Groups were compared using
ANOVA followed by Tukey's test for multiple comparisons. The level of significance was set at $P<0.05$.

\section{Results}

3.1. Prostate Weight of the $B P H$ and $B P H+S E$ Groups. The mean prostate weight of the $\mathrm{BPH}$ group was significantly higher than that of the control group $(P<0.05)$. Compared with the BPH groups, administration of SE led to significant reduction in prostate weight $(P<0.05)$ (Table 2$)$. The reduction in prostate weight was proportional to the increase in the dose of SE applied.

3.2. Pathohistological Findings in Each Experimental Group. In the control group, one layer of low-columnar epithelial cells formed a secretory lumen that was filled with thin acidophilic materials. Undeveloped epithelial cells forming the prostate gland were arranged as a single layer (Figure 2(a)). In contrast, the epithelial cells in the BPH group were arranged in several uneven layers, and the gland was excessively developed (Figure 2(b)). In the BPH + SE1 group, columnar epithelial cells were arranged as multiple layers, and the proliferation of epithelial cells and the number of glands were increased compared with the normal group. However, in comparison with the $\mathrm{BPH}$ group, prostate cell proliferation and the development of glands were noticeably decreased (Figure 2(c)). It was difficult to find a histological difference between the control group and the $\mathrm{BPH}+\mathrm{SE} 2$ group (Figure 2(d)).

3.3. Comparison of Oxidative Stress in the Serum and Prostate. Oxidative stress was assessed by measurement of SOD activity in the serum as well as the level of 8 -OHdG in prostate tissues. A significant increase in oxidative stress was found in the BPH group compared with the control group $(P<0.05)$. 
TABLE 2: Comparison of parameters in each group.

\begin{tabular}{lcccccc}
\hline & & \multicolumn{2}{c}{ Oxidative stress } & Apoptosis & Activity of 5-alpha reductase \\
& Prostate weight (g) & $\begin{array}{c}\text { Activity of SOD } \\
\text { in serum (\%) }\end{array}$ & $\begin{array}{c}\text { Concentration of } \\
\text {-OHdG in prostate } \\
(\mathrm{ng} / \mathrm{mL})\end{array}$ & $\begin{array}{c}\text { Concentration of } \\
\text { caspase-3 in prostate } \\
(\mathrm{ug} / \mathrm{mL})\end{array}$ & $\begin{array}{c}\text { In serum (\%) } \\
\text { In prostate (\%) }\end{array}$ \\
\hline Control & $1.040 \pm 0.061$ & $35.761 \pm 2.115$ & $0.620 \pm 0.061$ & $3.661 \pm 0.053$ & $5.668 \pm 0.292$ & $7.178 \pm 0.092$ \\
BPH & $1.421 \pm 0.039^{*}$ & $81.345 \pm 0.892^{*}$ & $7.651 \pm 0.306^{*}$ & $24.456 \pm 1.625^{*}$ & $18.863 \pm 0.648^{*}$ & $27.176 \pm 0.719^{*}$ \\
BPH + SE1 & $0.988 \pm 0.040^{* *}$ & $62.047 \pm 0.875^{* *}$ & $6.493 \pm 0.253^{* *}$ & $17.212 \pm 0.787^{* *}$ & $11.515 \pm 0.344^{* *}$ & $18.177 \pm 0.643^{* *}$ \\
BPH + SE2 & $0.878 \pm 0.068^{* *}$ & $55.816 \pm 1.488^{* *}$ & $4.060 \pm 0.355^{* *}$ & $12.975 \pm 0.703^{* *}$ & $11.347 \pm 0.120^{* *}$ & $15.453 \pm 0.382^{* *}$ \\
\hline
\end{tabular}

The mean prostate weight of the BPH group was significantly higher than that of the control group, whereas the means of the BPH + SE groups were significantly reduced compared to the $\mathrm{BPH}$ group.

A significant increase in oxidative stress was found in the BPH group compared with the control group, and oxidative stress was significantly reduced in the two $\mathrm{BPH}+\mathrm{SE}$ groups.

A significant increase in the concentration of caspase-3 was found in the BPH group compared with the control group, and it was significantly decreased in the two BPH + SE groups.

A significant increase in 5-alpha reductase activity was found in the BPH group compared with the control group, and it was significantly reduced in the two $\mathrm{BPH}+\mathrm{SE}$ groups.

BPH: benign prostatic hyperplasia group; BPH + SE1: BPH group treated with Seoritae extract $(228 \mathrm{mg} / \mathrm{kg}) ; \mathrm{BPH}+\mathrm{SE2}$ : BPH group treated with Seoritae extract (457 mg/kg); SOD: superoxide dismutase; 8-OHdG: 8-hydroxy-2-deoxyguanosine.

${ }^{*}$ Significant difference $(P<0.05)$ compared with the control group.

${ }^{* *}$ Significant difference $(P<0.05)$ compared with the $\mathrm{BPH}$ group.

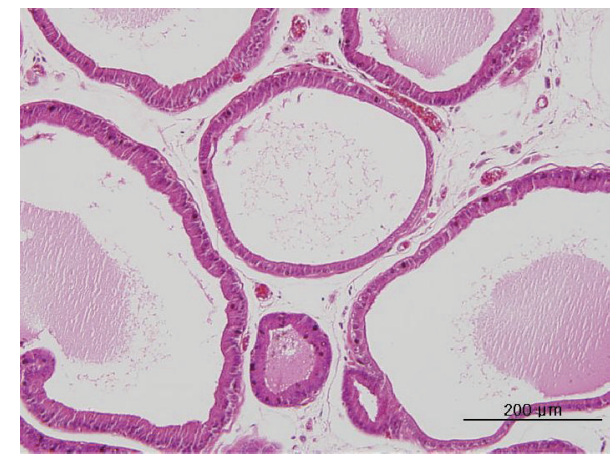

(a)

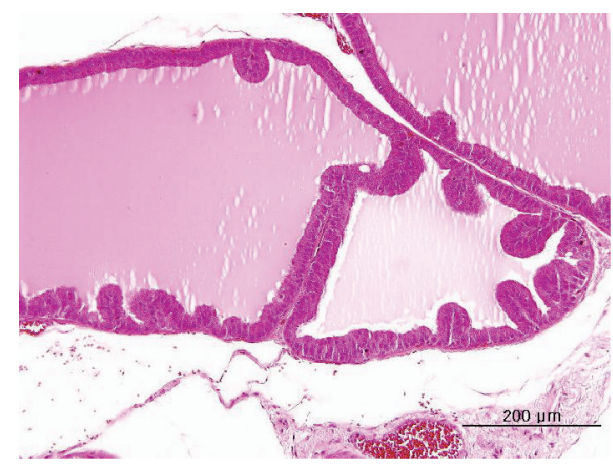

(c)

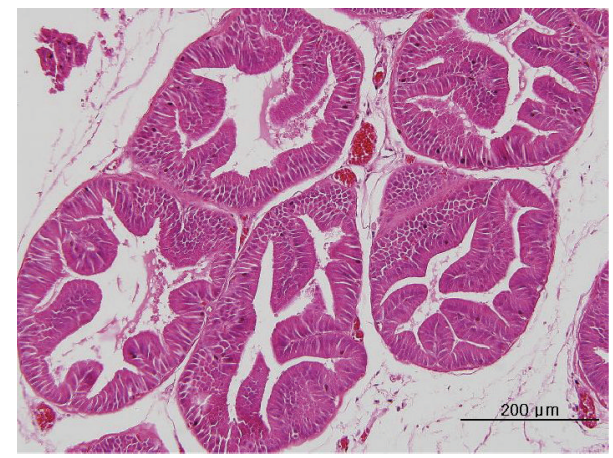

(b)

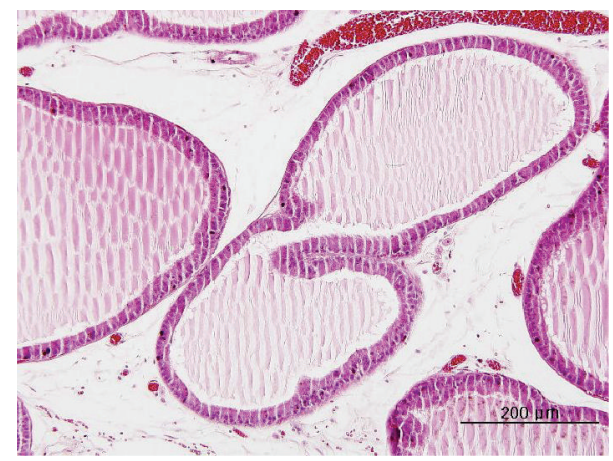

(d)

FIGURE 2: Histological analysis of prostate specimens taken from each experimental group. (a) Secretory luminal cells were lined with a single layer of low columnar epithelium and the acini were filled with pale eosinophilic materials (H \& E stain, $\times 200)$. (b) The epithelial cells in glands were arranged as several uneven layers and the gland was excessively developed (H \& E stain, $\times 200)$. (c) In comparison to (b), the proliferation of columnar epithelial cells in the BPH + SE1 group was restricted, and the development of the gland was limited (H \& E stain, $\times 200$ ). (d) It is difficult to find a difference between (d) and (a), except for the multiple layers of columnar epithelial cells in some parts of the gland in (d). H \& E: hematoxylin and eosin; (a) control; (b) BPH; (c) BPH + SE1; (d) BPH + SE2. 
In the two $\mathrm{BPH}+\mathrm{SE}$ groups, however, oxidative stress was significantly reduced compared to the $\mathrm{BPH}$ group $(P<0.05)$ (Table 2). The reduction of oxidative stress was proportional to the increase in the dose of SE applied.

3.4. Comparison of Apoptosis. A significant increase in the concentration of caspase- 3 was found in the BPH group when compared with the control group $(P<0.05)$. In the $\mathrm{BPH}+\mathrm{SE}$ groups, however, the concentration of caspase- 3 was significantly reduced compared to the $\mathrm{BPH}$ group $(P<$ $0.05)$ and significantly increased compared with the control group $(P<0.05)$ (Table 2$)$.

3.5. Comparison of 5-Alpha Reductase Activity in the Serum and Prostate Tissues. A significant increase in 5-alpha reductase activity was found in the serum and prostate samples from the $\mathrm{BPH}$ group when compared to the control group $(P<0.05)$. On the other hand, in the $\mathrm{BPH}+\mathrm{SE}$ groups, the activity of 5-alpha reductase was reduced in comparison to the BPH group $(P<0.05)$ (Table 2$)$. However, the reduction in 5-alpha reductase activity was not proportional to the increase in SE dose. There was no significant difference in the 5 -alpha reductase activity in the sera taken from the $\mathrm{BPH}+\mathrm{SE}$ groups $(P>0.05)$. In prostate tissue, the $\mathrm{BPH}+\mathrm{SE} 1$ group showed an increase in 5-alpha reductase activity in comparison to the $\mathrm{BPH}+\mathrm{SE} 2$ groups $(P<0.05)$.

\section{Discussion}

The molecular biological mechanisms influencing the etiology of BPH have not been elucidated; however, BPH has a high incidence among elderly males [16], and aging is known to be a risk factor for developing BPH [17]. The free radical theory suggests that free radicals generated in the human body during the process of producing energy lead to oxidative injury of cell components such as proteins, DNA, and lipids. This results in the loss of tissue and organ functions as well as cell death $[18,19]$ and is related to the occurrence and progression of BPH. In fact, a correlation between oxidative stress and $\mathrm{BPH}$ occurrence has been identified in several studies $[3,4]$, and we therefore hypothesized that the oxidative stress mechanism is related to the occurrence and progression of $\mathrm{BPH}$. Moreover, anthocyanin, as a potent antioxidant, was found to be effective in decreasing prostate volume and preventing the progression of $\mathrm{BPH}$ in our previous report [5]. In addition, the presence of testes producing androgens is a known risk factor for $\mathrm{BPH}$ since androgens, including testosterone and dihydrotestosterone (DHT), play a central role in regulating cell proliferation and death in the prostate. In particular, the expression of DHT, which is the most active form of testosterone that is converted from testosterone by 5 -alpha reductase, is correlated with the occurrence and progression of $\mathrm{BPH}[2]$.

Considering the above, we hypothesized that treatment with a compound that inhibits the conversion of testosterone to DHT and decreases oxidative stress would prevent the occurrence and progression of $\mathrm{BPH}$. The main findings of the present study were as follows: (1) BPH was induced by injection of testosterone enanthate, and prostate weight was decreased as a result of the SE treatment; (2) oxidative stress in the serum and the prostate was increased by induction of BPH and was reduced by SE treatment; (3) expression of caspase-3, which is a measure of apoptosis, was increased in rats with $\mathrm{BPH}$ and decreased by the SE treatment; (4) the activity of 5-alpha-reductase was increased due to the induction of BPH but was decreased as a result of the SE supplementation.

In our study, the HPLC chromatogram revealed that SE was composed of variable isoflavones and anthocyanins (Table 1). Therefore, the effect of SE was based on the properties of these isoflavones and anthocyanins. Based on our results, we hypothesized that the occurrence and progression of $\mathrm{BPH}$ were related to the activity of 5-alpha reductase and the cellular damage by oxidative stress. We also suggest that the reduction in prostate weight after the administration of SE might be due to the inhibitory effect of 5-alpha reductase as well as the antioxidant properties of the isoflavones and anthocyanin in SE.

Oxidative stress reflects an imbalance between the systemic manifestation of reactive oxygen species (ROS) and a biological system's ability to readily detoxify the reactive intermediates or to repair the resulting damage. Disturbances in the normal redox state of cells can cause toxic effects through the production of peroxides and free radicals that damage all components of the cell including proteins, lipids, and DNA. This is thought to result in the loss of cellular and tissue function.

In our study, oxidative stress was increased in the serum and prostate as a result of $\mathrm{BPH}$, which was confirmed by measuring the activity of SOD in the serum and the level of 8-OHdG in the prostate. In addition, these changes were reversed after the administration of SE. Increased oxidative stress might cause oxidative injury to various cell cycle control proteins and disrupt the balance between proliferation and cell death in the prostate, resulting in the occurrence and progression of $\mathrm{BPH}$.

It is known that genistein and daidzein, two types of isoflavones, inhibit hydrogen peroxide production and superoxide anion generation in cells, possibly via indirect regulation of antioxidant enzyme levels and isoflavones. Reduced derivatives of these compounds can also inhibit microsomal lipid peroxidation in vitro $[7,20]$. Furthermore, anthocyanin leads to the removal of superoxide, singlet oxygen, hydrogen peroxide, and hydroxyl radicals and also stabilizes and inactivates free radicals and prevents cellular oxidative stress [21].

We suggest that the improvement of oxidative stress after the administration of SE in our study was due to the antioxidant effects of the isoflavones and anthocyanins. This effect might have decreased the oxidative injury to the prostate cells and prevented abnormal cell proliferation, thus restoring the balance between proliferation and apoptosis in BPH.

Interestingly, the BPH group showed a markedly increased concentration of caspase-3, the final effector of cell lysis, compared to that observed in the control group. Similar results were reported in several studies [22-25]. Kosova et al. reported that a considerable degree of oxidative stress 
and DNA damage occurs in BPH (although less than that occurring in prostate cancer) and that levels of caspase-3 are higher in $\mathrm{BPH}$ than in prostate cancer [25]. In addition, Shariat et al. reported that levels of Bcl-2, Bax, Ki-67, and caspase-3 were highly elevated in patients with $\mathrm{BPH}$ when compared to patients with a normal prostate [24]. According to the above reports and our result, we suggest that elevated caspase- 3 in $\mathrm{BPH}$ might be a homeostatic reaction that balances the apoptosis-proliferation equilibrium, which occurs due to abnormal hyperproliferation of prostate cells induced by DNA damage from oxidative stress.

In contrast, the concentration of caspase- 3 in $\mathrm{BPH}+\mathrm{SE}$ groups was markedly increased compared with that of the control group and was significantly decreased compared with that of the BPH group. We suggest that the number of cells involved in apoptosis in $\mathrm{BPH}+\mathrm{SE}$ groups may have been decreased compared to that in the BPH group. This is because cellular damage from oxidative stress, which leads to abnormal prostate cell hyperproliferation, might be decreased by antioxidant effect of SE. Thus, the homeostatic reaction that balances the apoptosis-proliferation equilibrium in the $\mathrm{BPH}+$ SE groups might have been decreased relatively to the $\mathrm{BPH}$ group. This might have resulted in a significant decrease in the concentration of caspase- 3 in $\mathrm{BPH}+\mathrm{SE}$ groups when compared to the $\mathrm{BPH}$ group.

It is well known that isoflavones have the ability to block the overproduction of certain steroid hormones that influence the promotion and progression of BPH. Specifically, isoflavones are known to inhibit 5-alpha reductase, aromatase, and 17 beta-hydroxysteroid dehydrogenase enzymes, which block the synthesis of DHT, estrone, and other steroid hormones, respectively. DHT is known to enhance prostate cell division and is directly linked to the promotion and progression of $\mathrm{BPH}[2,10,26]$. In our study, 5-alpha reductase activity was increased in the $\mathrm{BPH}$ group and decreased after administration of SE. These results demonstrated that isoflavones inhibit 5-alpha reductase and suggest that this inhibitory effect may be one of the main mechanisms by which SE decreases prostate weight and prevents the progression of $\mathrm{BPH}$.

We note that our study has several limitations. First, there are many proposed mechanisms by which isoflavones might induce their therapeutic effects, including inhibition of protein tyrosine kinase [6], binding to estrogen receptors [27], inhibition of ROS production [7, 8], inhibition of angiogenesis [28], modulation of sex steroid binding proteins [29], and inhibition of 5 alpha-reductase activity [6]. Among these possibilities, we suggest that the antioxidant effect and the inhibition of 5-alpha reductase are the main mechanisms involved in the SE-induced decrease in prostate weight. However, it is possible that other mechanisms may contribute to the antioxidant effects of isoflavone and suggest that further study on the relevance between these mechanisms and the decrease in the prostate weight after SE administration of SE should be conducted.

Second, our results suggested that the antioxidant effect of anthocyanin contributed to the reduction in prostate weight in this study. It is well established that anthocyanin is a strong antioxidant [21] and effective in reducing prostate weight in an animal model of BPH [5]; however, the anthocyanin content in SE was found to be considerably low. Therefore, the contribution of anthocyanin in relation to our results will need to be assessed further.

\section{Conclusions}

Administration of $\mathrm{SE}$ to rats during induction of $\mathrm{BPH}$ resulted in a reduction in prostate weight, oxidative stress, and 5-alpha reductase activity, as well as a decrease in apoptosis. We believe that the occurrence and progression of $\mathrm{BPH}$ are related to an oxidative stress-mediated mechanism and increasing activity of 5-alpha reductase. Also we believed that the antioxidant properties and inhibitory effect of 5-alpha reductase of isoflavones and anthocyanin in SE would be effective in treating $\mathrm{BPH}$. We therefore suggest that SE has the potential to replace or improve current BPH treatments.

\section{Conflict of Interests}

The authors declare that there is no conflict of interests regarding the publication of this paper.

\section{Acknowledgment}

This work was supported by a Grant from the NextGeneration Biogreen 21 Program (no. PJ009546), Rural Development Administration, Republic of Korea.

\section{References}

[1] E. Arias, "United States life tables, 2007," National Vital Statistics Reports, vol. 59, no. 9, pp. 1-60, 2011.

[2] C. Carson III and R. Rittmaster, "The role of dihydrotestosterone in benign prostatic hyperplasia," Urology, vol. 61, no. 4, pp. 2-7, 2003.

[3] M. Aryal, A. Pandeya, N. Gautam et al., "Oxidative stress in benign prostate hyperplasia," Nepal Medical College Journal, vol. 9, no. 4, pp. 222-224, 2007.

[4] A. Aydin, Z. Arsova-Sarafinovska, A. Sayal et al., "Oxidative stress and antioxidant status in non-metastatic prostate cancer and benign prostatic hyperplasia," Clinical Biochemistry, vol. 39, no. 2, pp. 176-179, 2006.

[5] H. Jang, U.-S. Ha, S.-J. Kim et al., "Anthocyanin extracted from black soybean reduces prostate weight and promotes apoptosis in the prostatic hyperplasia-induced rat model," Journal of Agricultural and Food Chemistry, vol. 58, no. 24, pp. 1268612691, 2010.

[6] F. H. Sarkar and Y. Li, "Soy isoflavones and cancer prevention," Cancer Investigation, vol. 21, no. 5, pp. 744-757, 2003.

[7] R. P. Patel, B. J. Boersma, J. H. Crawford et al., "Antioxidant mechanisms of isoflavones in lipid systems: paradoxical effects of peroxyl radical scavenging," Free Radical Biology and Medicine, vol. 31, no. 12, pp. 1570-1581, 2001.

[8] M. I. Yousef, K. I. Kamel, A. M. Esmail, and H. H. Baghdadi, "Antioxidant activities and lipid lowering effects of isoflavone in male rabbits," Food and Chemical Toxicology, vol. 42, no. 9, pp. 1497-1503, 2004.

[9] R. Bergan, E. Kyle, P. Nguyen, J. Trepel, C. Ingui, and L. Neckers, "Genistein-stimulated adherence of prostate cancer 
cells is associated with the binding of focal adhesion kinase to beta-1-integrin," Clinical and Experimental Metastasis, vol. 14, no. 4, pp. 389-398, 1996.

[10] J. G. Lewis, S. Nakajin, S. Ohno, A. Warnock, C. M. Florkowski, and P. A. Elder, "Circulating levels of isoflavones and markers of $5 \alpha$-reductase activity are higher in Japanese compared with New Zealand males: what is the role of circulating steroids in prostate disease?” Steroids, vol. 70, no. 14, pp. 974-979, 2005.

[11] B. A. J. Evans, K. Griffiths, and M. S. Morton, "Inhibition of $5 \alpha$-reductase in genital skin fibroblasts and prostate tissue by dietary lignans and isoflavonoids," Journal of Endocrinology, vol. 147, no. 2, pp. 295-302, 1995.

[12] R. Schindler and R. Mentlein, "Flavonoids and vitamin E reduce the release of the angiogenic peptide vascular endothelial growth factor from human tumor cells," Journal of Nutrition, vol. 136, no. 6, pp. 1477-1482, 2006.

[13] D. Bagchi, C. K. Sen, M. Bagchi, and M. Atalay, "Antiangiogenic, antioxidant, and anti-carcinogenic properties of a novel anthocyanin-rich berry extract formula," Biochemistry, vol. 69, no. 1, pp. 75-80, 2004.

[14] M. Liu, X. Q. Li, C. Weber, C. Y. Lee, J. Brown, and R. H. Liu, "Antioxidant and antiproliferative activities of raspberries," Journal of Agricultural and Food Chemistry, vol. 50, no. 10, pp. 2926-2930, 2002.

[15] Z. Liu, J. Schwimer, D. Liu, F. L. Greenway, C. T. Anthony, and E. A. Woltering, "Black raspberry extract and fractions contain angiogenesis inhibitors," Journal of Agricultural and Food Chemistry, vol. 53, no. 10, pp. 3909-3915, 2005.

[16] J. T. Isaacs and D. S. Coffey, "Etiology and disease process of benign prostatic hyperplasia," Prostate, vol. 15, no. 2, pp. 33-50, 1989.

[17] D. S. Coffey and P. C. Walsh, "Clinical and experimental studies of benign prostatic hyperplasia," Urologic Clinics of North America, vol. 17, no. 3, pp. 461-475, 1990.

[18] M. de la Fuente, "Effects of antioxidants on immune system ageing," European Journal of Clinical Nutrition, vol. 56, no. 3, pp. S5-S8, 2002.

[19] D. Harman, "Aging: a theory based on free radical and radiation chemistry," Journal of Gerontology, vol. 11, no. 3, pp. 298-300, 1956.

[20] H. Wei, R. Bowen, Q. Cai, S. Barnes, and Y. Wang, "Antioxidant and antipromotional effects of the soybean isoflavone genistein," Proceedings of the Society for Experimental Biology and Medicine, vol. 208, no. 1, pp. 124-130, 1995.

[21] A. Solomon, S. Golubowicz, Z. Yablowicz et al., "Antioxidant activities and anthocyanin content of fresh fruits of common fig (Ficus carica L.)," Journal of Agricultural and Food Chemistry, vol. 54, no. 20, pp. 7717-7723, 2006.

[22] A. J. O’Neill, S. A. Boran, C. O’Keane et al., "Caspase 3 expression in benign prostatic hyperplasia and prostate carcinoma," Prostate, vol. 47, no. 3, pp. 183-188, 2001.

[23] L. Wang, J.-R. Yang, L.-Y. Yang, Z.-T. Liu, J.-M. Rao, and L.-F. Liu, "Expression of Ki-67, Bcl-2, Bax and caspase-3 in benign prostatic hyperplasia combined with prostatitis and their significances," Journal of Central South University, vol. 33, no. 3, pp. 222-226, 2008.

[24] S. F. Shariat, R. Ashfaq, C. G. Roehrborn, K. M. Slawin, and Y. Lotan, "Expression of survivin and apoptotic biomarkers in benign prostatic hyperplasia," Journal of Urology, vol. 174, no. 5, pp. 2046-2050, 2005.
[25] F. Kosova, G. Temeltas, Z. Ari, and M. Lekili, "Possible relations between oxidative damage and apoptosis in benign prostate hyperplasia and prostate cancer patients," Tumour Biology, 2013.

[26] J. D. Wilson, "Role of dihydrotestosterone in androgen action," The Prostate, vol. 6, pp. 88-92, 1996.

[27] T. Hong, T. Nakagawa, W. Pan et al., "Isoflavones stimulate estrogen receptor-mediated core histone acetylation," Biochemical and Biophysical Research Communications, vol. 317, no. 1, pp. 259-264, 2004.

[28] S.-J. Su, T.-M. Yeh, W.-J. Chuang et al., "The novel targets for anti-angiogenesis of genistein on human cancer cells," Biochemical Pharmacology, vol. 69, no. 2, pp. 307-318, 2005.

[29] A. M. Pino, L. E. Valladares, M. A. Palma, A. M. Mancilla, M. Yáñez, and C. Albala, "Dietary isoflavones affect sex hormonebinding globulin levels in postmenopausal women," Journal of Clinical Endocrinology and Metabolism, vol. 85, no. 8, pp. 27972800, 2000. 


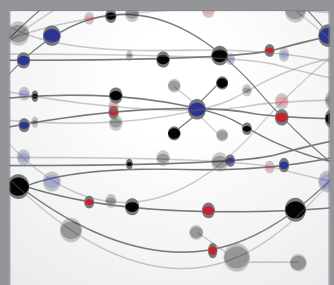

The Scientific World Journal
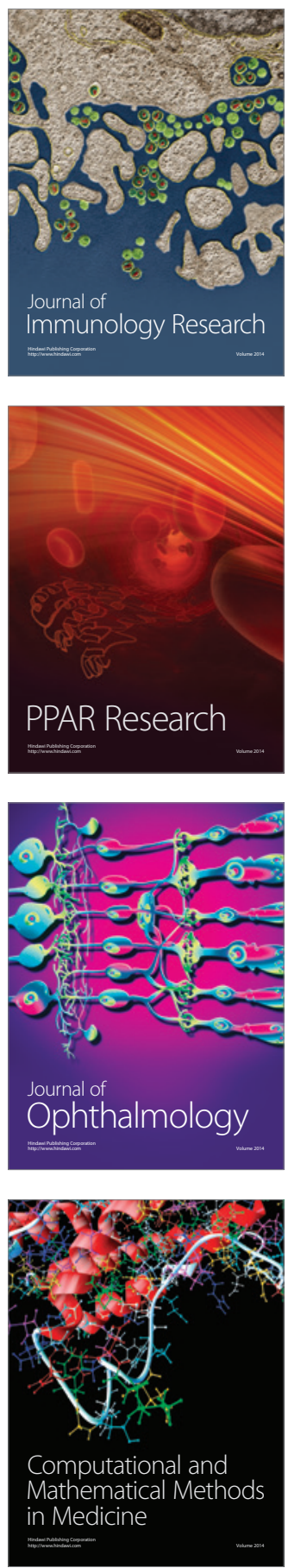

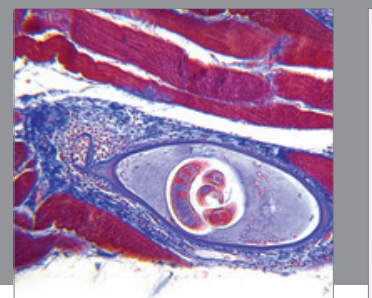

Gastroenterology

Research and Practice
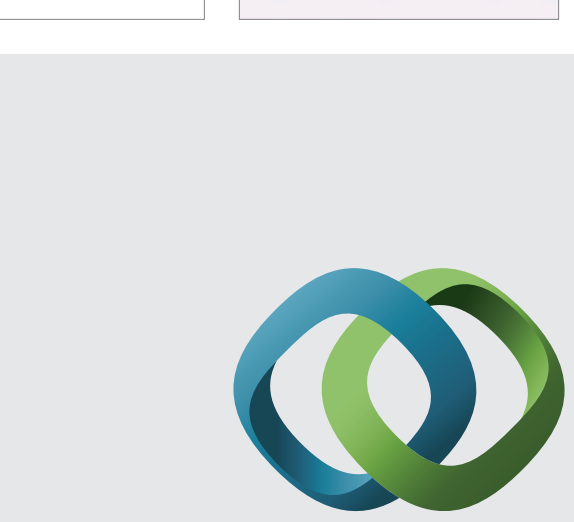

\section{Hindawi}

Submit your manuscripts at

http://www.hindawi.com
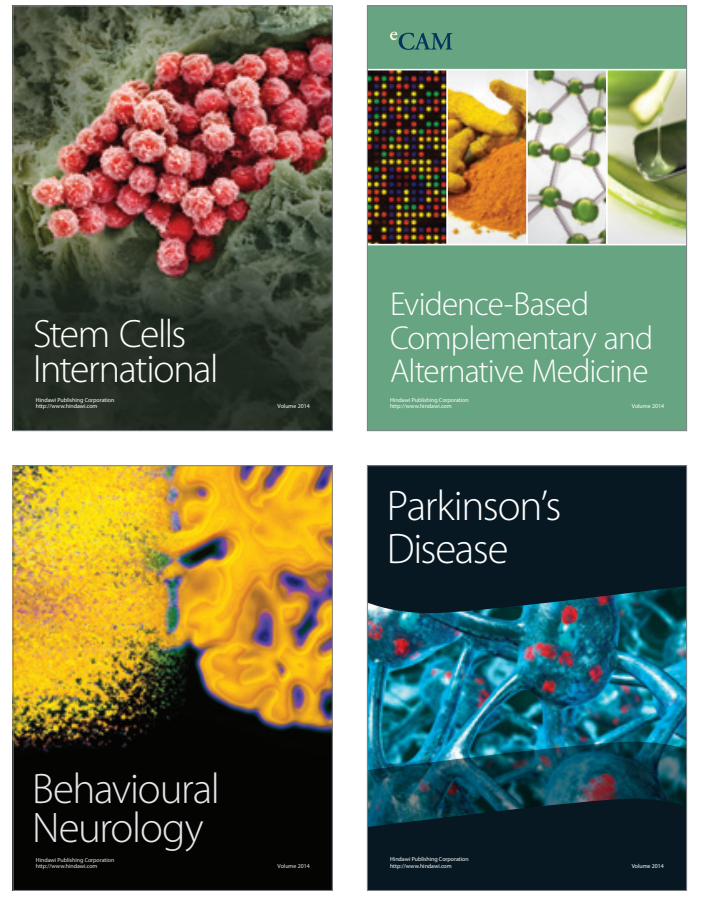
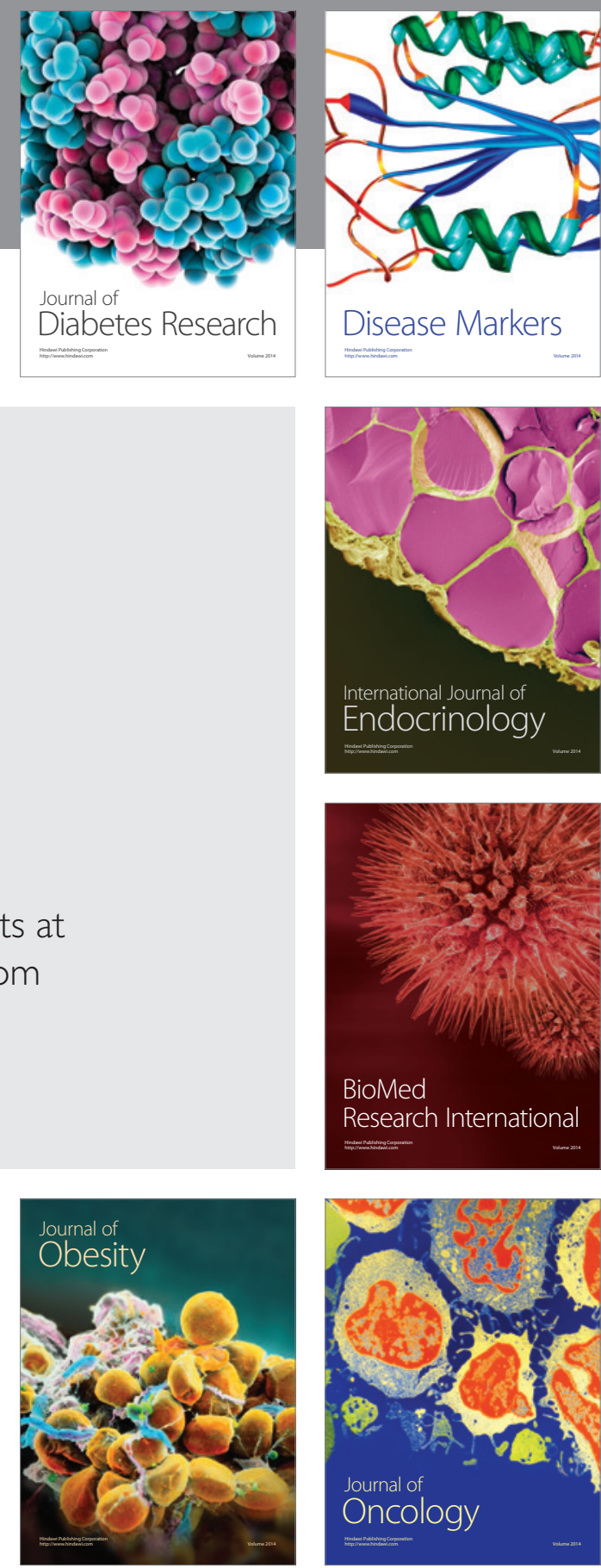

Disease Markers
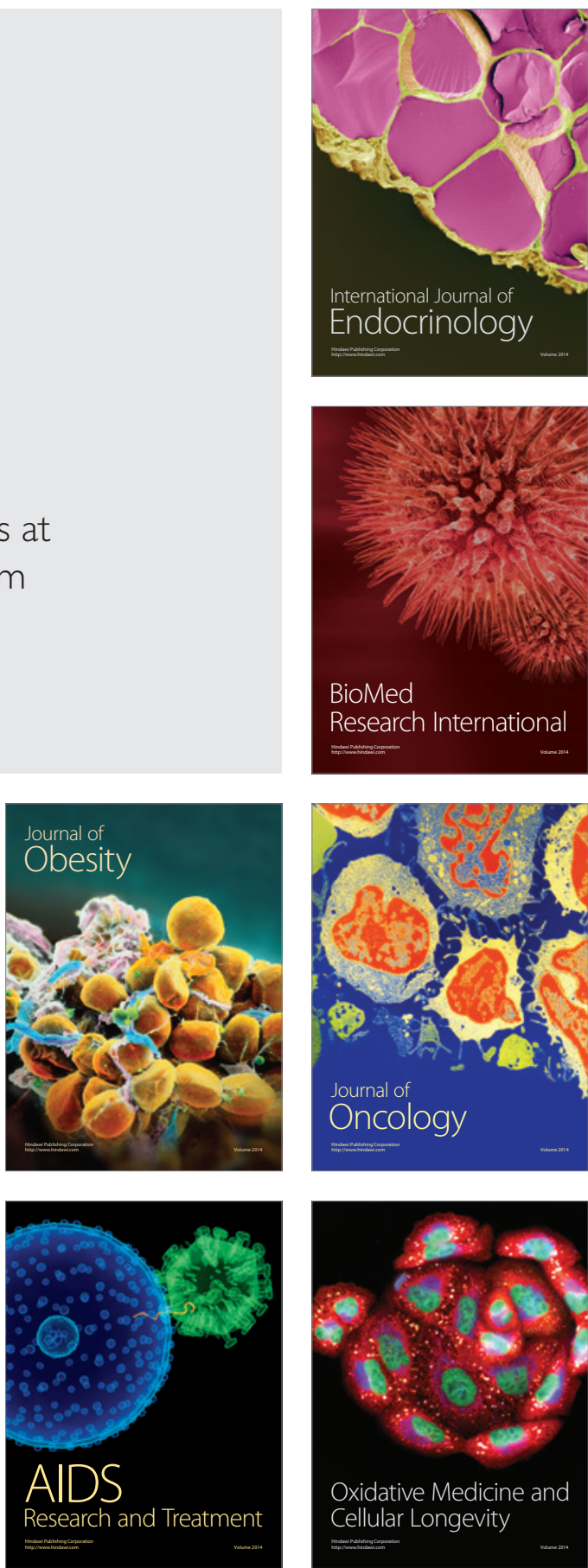